\title{
Effect of a Radical Scavenger "Water Soluble Protein" from Broad Beans (Vicia faba) on Antioxidative Enzyme Activity in Cellular Aging
}

\author{
Yoshinori OKADA ${ }^{1, *}$ and Mizue OKADA ${ }^{2}$ \\ ${ }^{1}$ Laboratory on Ageing, Chukyo Junior College, 2216, Toki-Cho, Mizunami, \\ Gifu 509-6192, Japan \\ ${ }^{2}$ Department of Hygiene, Gifu University School of Medicine, 40 Tsukasa-machi, \\ Gifu 500-8076, Japan
}

(Received November 4, 1998)

\begin{abstract}
Summary We isolated a free-radical scavenger "water soluble protein (WSP)" from broad beans. Hydrocortisone (HC) is known to inhibit superoxide generation and was used as the reference scavenger. WSP was examined for its effect on antioxidation in young (PDL $20,25 \%$ of the maximum life span) and old (PDL 50, 62.5\% of the maximum life span) human fibroblasts (TIG-1). Cells were treated with WSP or HC for 4 and 6 wk in young cells, and for 3 and $6 \mathrm{wk}$ in old cells. The cytosolic superoxide dismutase activity in the cells treated with WSP or HC tended to decrease as compared with that in the non-treated cells (control) with the exception of WSP-treated young cells $4 \mathrm{wk}$ after culturing. Young cells were equal in glutathione peroxidase activity to the control, but the activity level in WSPor HC-treated young cells $6 \mathrm{wk}$ after culturing was 10-50\% lower than that in the control. Young and old cells treated with WSP or HC were superior to the control in catalase activity with the exception of HC-treated old cells. WSP- or HC-treated cells were higher in glutathione (GSH) concentration than the control with the exception of WSP-treated young cells $4 \mathrm{wk}$ after culturing and HC-treated old cells $6 \mathrm{wk}$ after culturing. Such increases in catalase activity and GSH concentration by WSP treatment may be related to the delay of cellular aging-dependent degeneration.
\end{abstract}

Key Words cellular aging, free-radical scavenger, antioxidative enzymes, broad bean protein, hydrocortisone

Harman $(1,2)$ has proposed that the accumulation of unrepaired oxidative damage may constitute an underlying cause of aging. There are several reports on agingdependent alterations in the antioxidative capacities of human diploid fibroblasts such as superoxide dismutase (SOD; EC 1.15.1.1) activity (3-5), catalase (EC 1.11.1.6) activity (6) and glutathione peroxidase (GSHPx; EC 1.11.1.9) activity (7) or reduced glutathione (GSH) concentration (7). Cytosol contains $\mathrm{Cu}$, Zn-SOD and GSH-Px with three-quarters as much as the total activity within the cell (8). SOD removes superoxide in a dismutation reaction that yields hydrogen peroxide and oxygen. Hydrogen peroxide is disposed of by GSH-Px or catalase. These enzymes constitute a defensive barrier of human fibroblast cells against oxidative damage.

Hydrocortisone (HC) has been shown to increase the cell density of fibroblasts at confluence (9). Fulder (10) also reported that the increase in density resulted from a faster growth rate. In addition, it was shown that HC inhibited superoxide generation (11).

The addition of antioxidants to culture media has been well examined (12-14). However, there is little information on the activities of antioxidative enzymes and the concentration of GSH in strains of human diploid

* To whom correspondence should be addressed. cells at stages after the addition of antioxidants.

We isolated a water-soluble protein capable of scavenging free radicals (WSP) from broad beans (Vicia faba) (15). The WSP exhibited a marked scavenging effect on superoxide as well as hydrogen peroxide. In addition, the WSP increased cellular growth to the same extent as HC (16). Therefore, we examined whether or not supplementation of human diploid fibroblasts with WSP can influence the antioxidative enzyme activities and the concentration of GSH. In the present study, we report the effect of WSP on cellular age, culture perioddependent oxidative stress and antioxidative enzyme activities in human diploid fibroblasts.

\section{MATERIALS AND METHODS}

Chemicals. Cytochrome $c$, xanthine oxidase, N-2-hydroxyethylpiperazine- $N^{\prime}$-2-ethanesulfonic acid (HEPES), and hydrogen peroxide were obtained from Sigma Chemical Co. (St. Louis, MO, U.S.A.). TIG-1-20 cells (JCRB0501) and TIG-1-50 cells (JCRB0504) were obtained from the Tokyo Metropolitan Institute of Gerontology (Tokyo, Japan). Fetal bovine serum (FBS) was purchased from Flow Laboratories (North Ryde, N.S.W., Australia), and trypsin from Difco Laboratories (Detroit, MI, U.S.A.). All other chemicals were purchased from Wako Pure Chemical Industries Ltd. 
(Osaka, Japan).

WSP preparation. WSP was prepared as described previously (15). Briefly, the purification procedures consist mainly of ammonium sulfate fractionation and gel filtration with Sephadex G-75. Gel filtration yielded two active fractions with molecular masses of 70 and $28 \mathrm{kDa}$. The $28 \mathrm{kDa}$ fraction was WSP, which was used as a free-radical scavenger in the following experiment.

Cell culture. Human lung fibroblasts (TIG-1 cells) were cultured as described previously (17). Briefly, the cells were cultured at $37^{\circ} \mathrm{C}$ in Eagle's minimum essential medium supplemented with $10 \%$ FBS, $28 \mathrm{~mm}$ HEPES and $1.5 \mu \mathrm{g} / \mathrm{mL}$ Fungizone under air- $\mathrm{CO}_{2}$ (95:5). Subculturing at $1 \times 10^{5}$ cells/dish was performed at intervals of approximately $1 \mathrm{wk}$ for young cells (TIG-1-20, population doubling level (PDL) 20, $25 \%$ of the maximum life span), or at intervals of approximately $10 \mathrm{~d}$ for old cells (TIG-1-50, PDL $50,62.5 \%$ of the maximum life span). The number of PDL achieved between subcultures was calculated by $\log _{2}$ (number of cells obtained at subculture/number of cells inoculated). The cumulative PDL was calculated as the sum of PDL achieved during each subculture. The culture media were supplemented for 4 or $6 \mathrm{wk}$ for young cells, and for 3 or $6 \mathrm{wk}$ for old cells, respectively, with W.SP at a final concentration of $1.25 \mu \mathrm{g} / \mathrm{mL}$ as a result of our preliminary experiment, or with $\mathrm{HC}$ at a final concentration of $5 \mu \mathrm{g} / \mathrm{mL}$ according to Fulder (10).

Effect of WSP on antioxidative enzyme activities and GSH concentration. Cytosolic antioxidative enzyme activities and cellular catalase activity were determined by use of WSP-treated cells which were prepared according to a modification of the methods of Rikans et al. (18) and Leist et al. (13), respectively.

Biochemical analysis. All enzyme assays were carried out on freshly prepared protein extracts. Cytosolic SOD activity was measured by the method of Crapo et al. (19) using a xanthine-xanthine oxidase system. Cytosolic GSH-Px activity was assayed according to the procedure of Tappel (8). Assay of catalase activity was due to the method of Chance and Maehly (20). The celIular GSH concentration was estimated according to modification of the method of Tietze (21). Protein was determined using bovine serum albumin as a standard by the method of Bradford (22).

Statistical analysis. Values were obtained as the means \pm SD in which comparison with the control was made by Student's $t$-test following ANOVA, and the difference was considered significant at $p<0.05$.

\section{RESULTS}

Antioxidative capacity of control cells

Young cell cultures started at PDL 20 and increased to PDL 37 and 44 after 4 and 6 wk, respectively. Old cell cultures started at PDL 50 and also increased to PDL 62 and 71 after 3 and $6 \mathrm{wk}$, respectively. Such increases in PDL per culture period were similarly observed in subsequent experiments.

Figure 1-a and b shows variation in the cytosolic SOD activity levels of young and old cells as a function of cul-

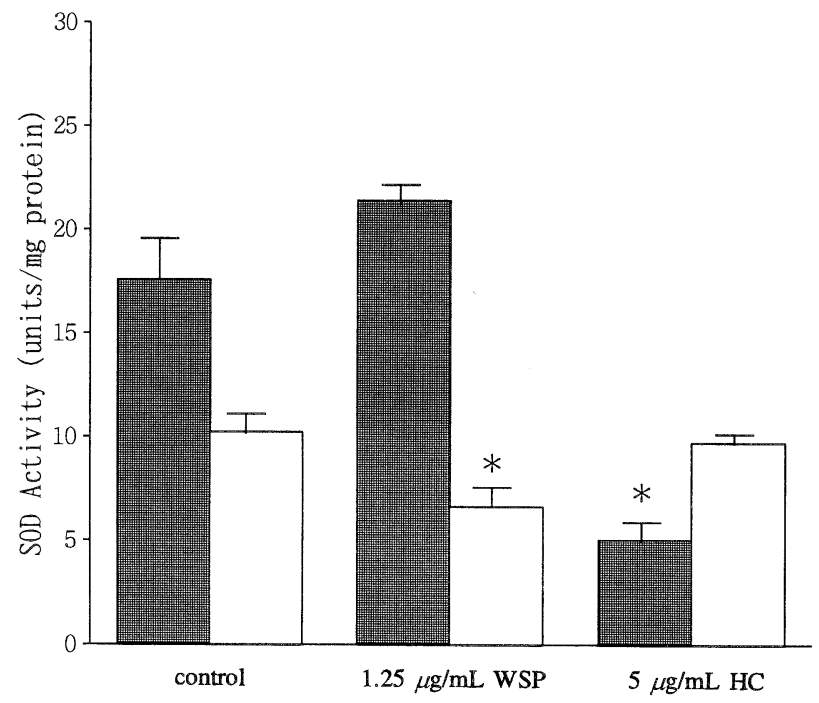

Fig. 1-a. Cytosolic SOD activities in young TIG-1 cells (PDL 20, 25\% of the maximum life span). The enzyme activities in 4 (PDL 37) and 6-wk cultures (PDL 44) are shown by shaded and open columns, respectively. Data are expressed as the means \pm standard deviation $(n=4)$ of three replicate analyses. One unit is defined as the amount of SOD required to inhibit the maximum rate of cytochrome $c$ reduction by $50 \%$. ${ }_{*} p<0.05$ compared with the control.

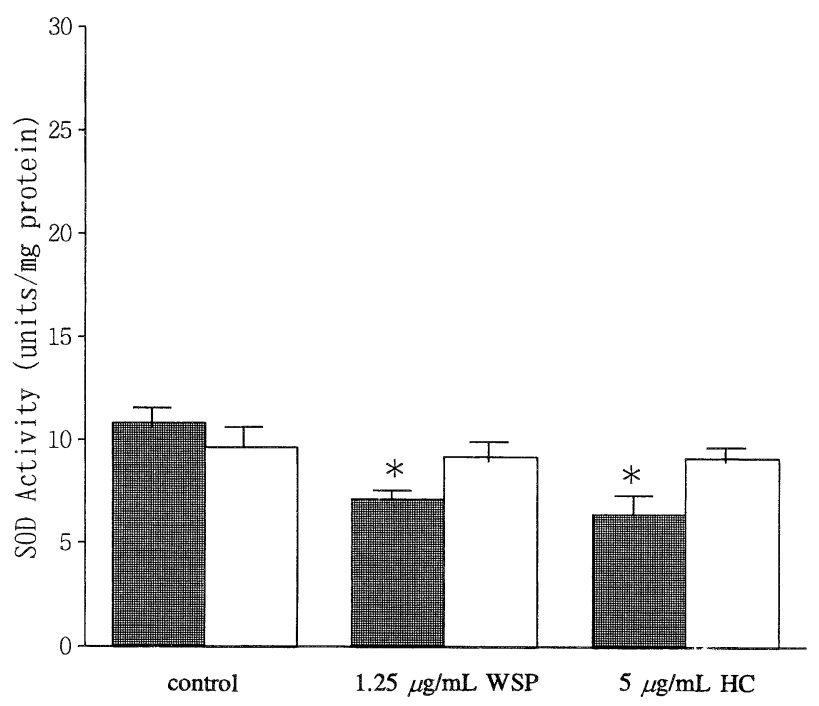

Fig. 1-b. Cytosolic SOD activities in old TIG-1 cells (PDL 50, 62.5\% of the maximum life span). The enzyme activities in 3 (PDL 62) and 6-wk cultures (PDL 71) are shown by shaded and open columns, respectively. Data are expressed as the means \pm standard deviation $(n=4)$ of three replicate analyses. The definition of one unit of SOD is the same as in Fig. 1-a. $* p<0.05$ compared with the control.

ture time. The SOD activity level of 6 -wk young cells (PDL 44) decreased by about $40 \%$ as compared with that of 4-wk cells (PDL 37) (Fig. 1-a). On the other hand, the SOD activity level in old cells remained constant throughout the culture period (from PDL 62 to 71) (Fig. 1-b). Although the SOD activity of young cells decreased with increasing culture periods (from PDL 37 


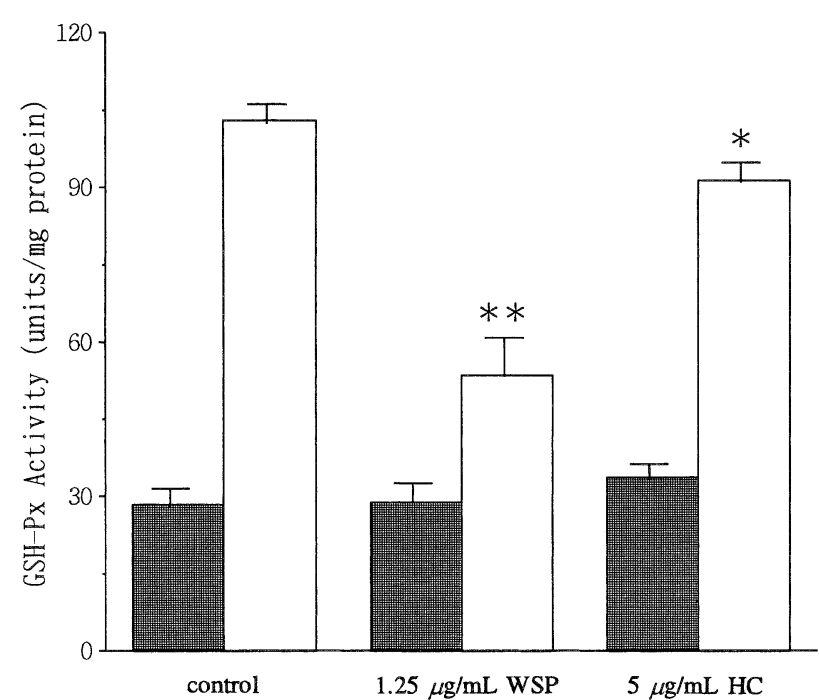

Fig. 2-a. Cytosolic GSH-Px activities in young TIG-1 cells (PDL 20, 25\% of the maximum life span). The enzyme activities in 4 (PDL 37) and 6-wk cultures (PDL 44) are shown by shaded and open columns, respectively. Data are expressed as the means \pm standard deviation $(n=4)$ of three replicate analyses. One unit is defined as the oxidation of 1 nmole of NADPH per minute. ${ }^{*} p<0.05,{ }^{* *} p<0.01$ compared with the control.

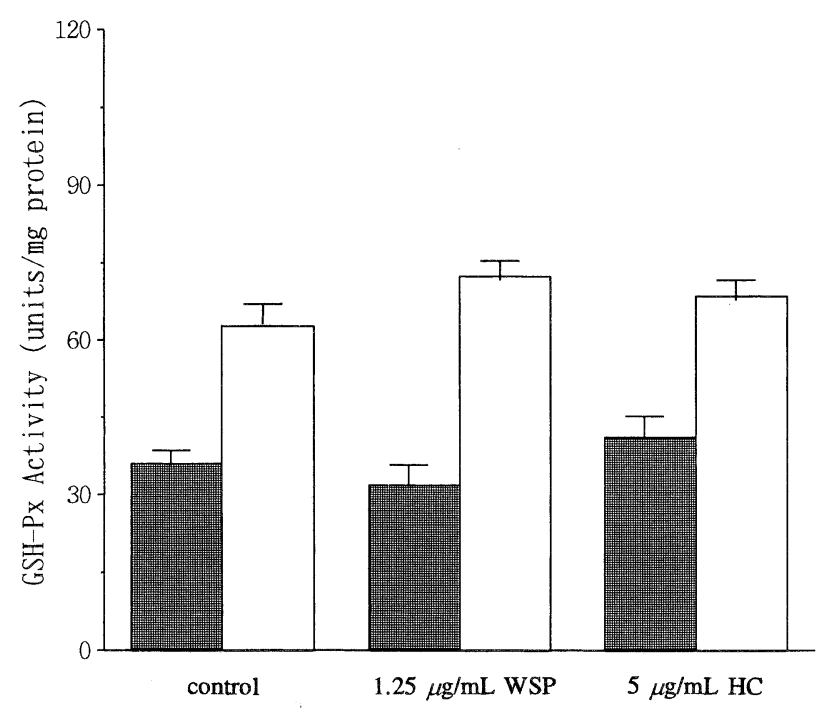

Fig. 2-b. Cytosolic GSH-Px activities in old TIG-1 cells (PDL 50, 62.5\% of the maximum life span). The enzyme activities in 3 (PDL 62) and 6-wk cultures (PDL 71) are shown by shaded and open columns, respectively. Data are expressed as the means \pm standard deviation $(n=4)$ of three replicate analyses. The definition of one unit of GSH-Px is the same as in Fig. 2-a. ${ }^{*} p<0.05$ compared with the control.

to 44), those of young (PDL 44) and old cells (PDL 71) in 6-wk cultures were at constant levels. In other words, their levels appeared unchangeable during cellular aging from PDL 44 to 71.

Figure 2-a and b shows variation in the cytosolic GSH-Px activity levels of young and old cells as a function of culture time. Its level of young cells cultured for $6 \mathrm{wk}$ (PDL 44) was about 4 times that for $4 \mathrm{wk}$ (PDL
37) (Fig. 2-a). Similarly, the level of old cells doubled in 6-wk cultures (PDL 71) relative to 3 -wk cultures (PDL 62 ), as shown in Fig. 2-b. The GSH-Px activity was 28.4 units/mg protein in young cells cultured for $4 \mathrm{wk}$ (PDL 37), while it was 36.0 units/mg protein in old cells cultured for $3 \mathrm{wk}$ (PDL 62). The GSH-Px activity seemed to increase slightly with advanced aging, but the activity level of old cells (PDL 71) was rather lower than that of young cells (PDL 44) in the same 6-wk cultures.

Figure 3 -a and $b$ shows variation in the catalase activity levels of young and old cells as a function of culture time. Both young and old cells cultured for $6 \mathrm{wk}$ were at activity levels half that of the cells cultured for 4 or $3 \mathrm{wk}$, although the level of old cells was somewhat higher as compared with that of the young cells.

Figure 4-a and b shows variation in GSH concentrations in young and old cells as a function of culture time. As for the GSH concentration in young cells, it was approximately 30\% lower in the cells cultured for 6 wk (PDL 44) as compared with that cultured for $4 \mathrm{wk}$ (PDL 37) (Fig. 4-a). The old cells cultured for $6 \mathrm{wk}$ (PDL 71) showed a $15 \%$ lower GSH level than those cultured for 3 wk (PDL 62) (Fig. 4-b).

Antioxidative capacity of WSP-or HC-treated cells

WSP or HC treatment affected the cytosolic SOD activities of young and old cells (Fig. 1-a, b). The SOD activity after 6 -wk culturing decreased by $40 \%$ in WSPtreated young cells as compared with the control, but its activity after 4-wk culturing tended to increase. On the other hand, the SOD activity of HC-treated young cells after 4 -wk culturing decreased by $70 \%$ as compared with that of the control, while the activity of $\mathrm{HC}$ treated cells after 6 -wk culturing was virtually equal to that of the control. The SOD activity of old cells treated with WSP or HC after 3-wk culturing decreased by 40$50 \%$ as compared with that of the control, but changes in the activity were not significant for WSP- or HCtreated cells after 6-wk culturing as compared to those of the control.

The cytosolic GSH-Px activity of young cells treated with WSP or HC after 6-wk culturing decreased by $10-50 \%$ as compared with that of the controls (Fig. 2a), but the activity levels of young cells treated with WSP or HC after 4-wk culturing were essentially identical to those of the controls. The levels of GSH-Px activity in WSP- or HC-treated old cells were similar to those of the controls (Fig. 2-b).

The catalase activity of young cells (Fig. 3-a) treated with WSP or HC increased by $60-600 \%$ as compared with that of the controls. Similarly, the activity of old cells (Fig. 3-b) treated with WSP increased by 30-45\% as compared with that of the controls, but there was no significant effect of $\mathrm{HC}$ on the activity in old cells.

The GSH concentration in young cells treated with WSP or HC increased as compared with that in the control with the exception of WSP-treated cells after 4-wk culturing, which was virtually equal to the level of the control (Fig. 4-a). Similarly, increases in the concentration were found in old cells treated with WSP or HC as 


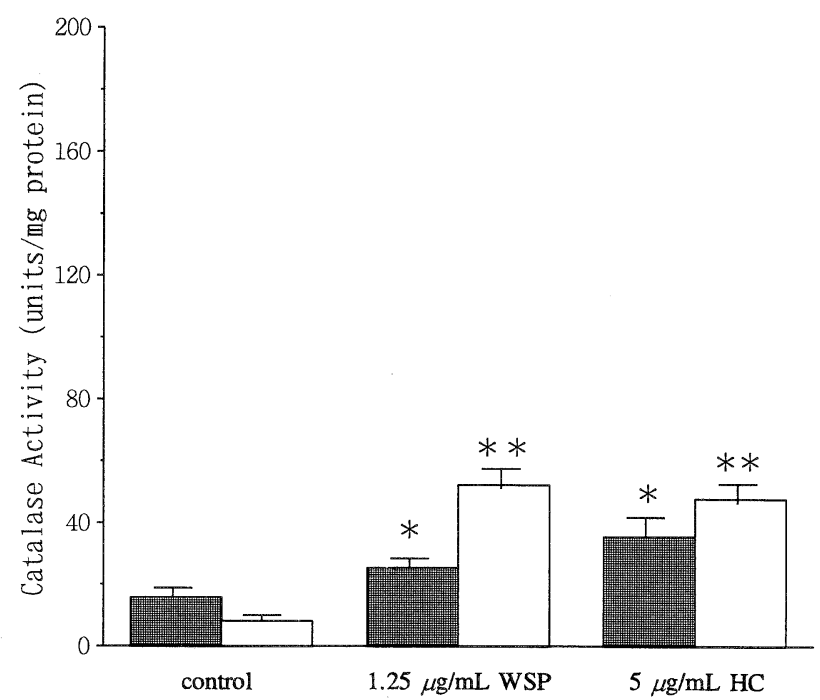

Fig. 3-a. Catalase activities in young TIG- 1 cells (PDL $20,25 \%$ of the maximum life span). The enzyme activities in 4 (PDL 37) and 6-wk cultures (PDL 44) are shown by shaded and open columns, respectively. Data are expressed as the means \pm standard deviation $(n=4)$ of three replicate analyses. One unit is defined as the removal of $1 \mu$ mole hydrogen peroxide per minute. ${ }^{*} p<0.05,{ }^{* *} p<0.01$ compared with the control.

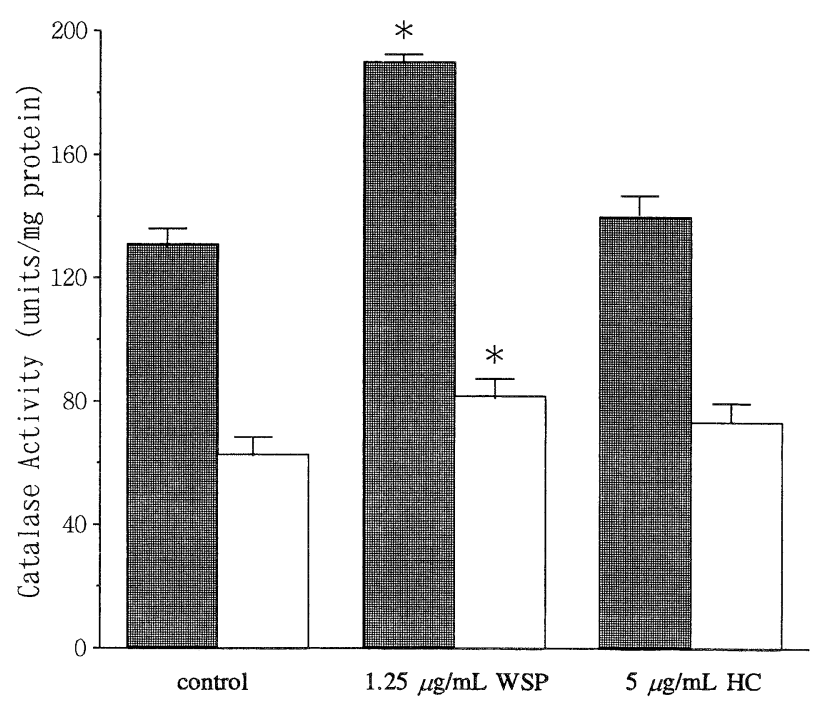

Fig. 3-b. Catalase activities in old TIG-1 cells (PDL 50, $62.5 \%$ of the maximum life span). The enzyme activities in 3 (PDL 62) and 6-wk cultures (PDL 71) are shown by shaded and open columns, respectively. Data are expressed as the means \pm standard deviation $(n=4)$ of three replicate analyses. The definition of one unit of catalase is the same as in Fig. 3 -a. ${ }^{*} p<0.05$ compared with the control.

compared to control samples, but the concentration of HC-treated cells after 6 -wk culturing was not significantly different from the control value (Fig. 4-b).

\section{DISCUSSION}

It has been reported that, during the in vitro aging of WI-38 human diploid fibroblasts, $\mathrm{Cu}, \mathrm{Zn}$-SOD activity

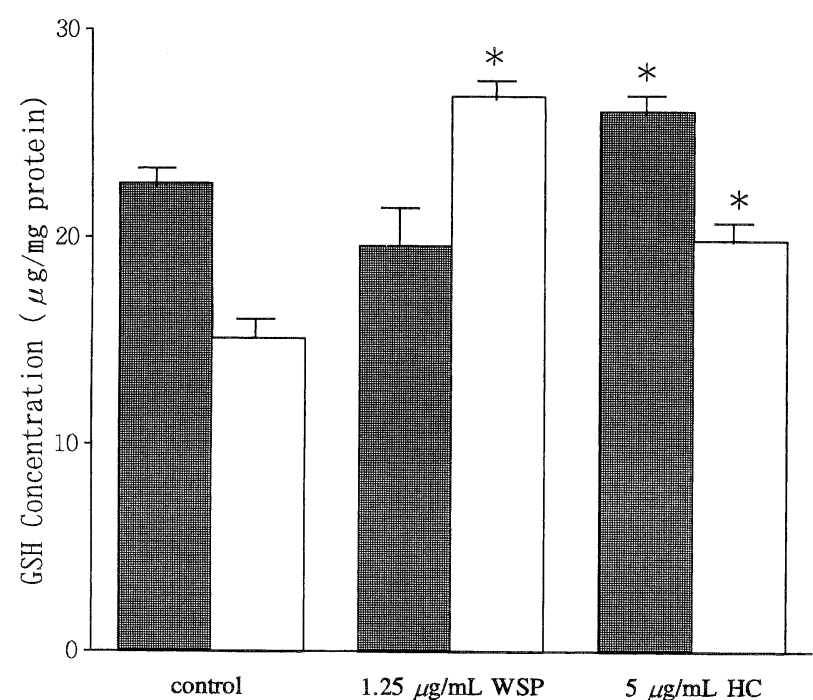

Fig. 4-a. Reduced glutathione concentrations in young TIG-1 cells (PDL 20, 25\% of the maximum life span). The enzyme activities in 4 (PDL 37) and 6-wk cultures (PDL 44) are shown by shaded and open columns, respectively. Data are expressed as the means \pm standard deviation $(n=4)$ of three replicate analyses. ${ }^{*} p<0.05$ compared with the control.

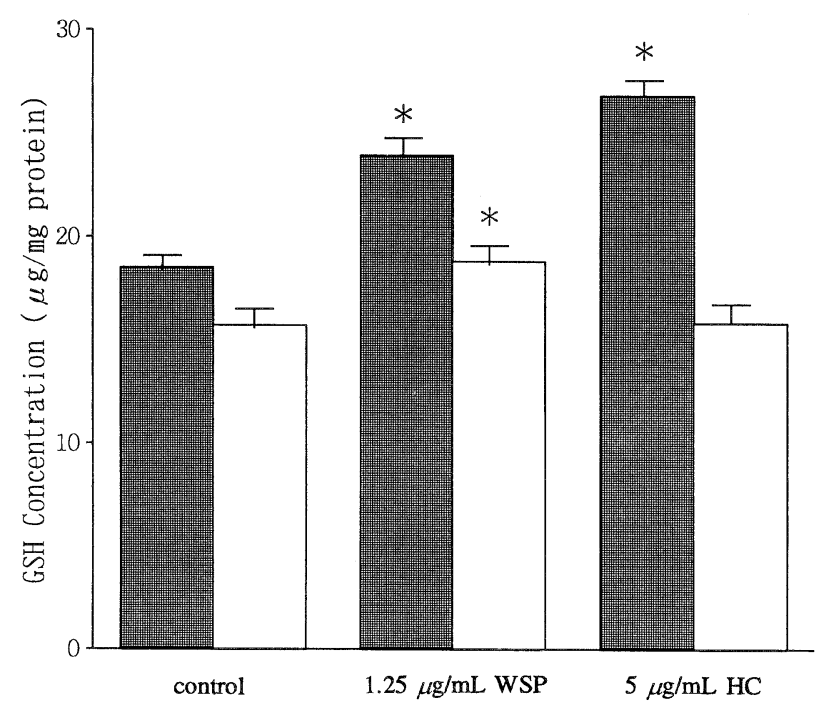

Fig. 4-b. Reduced glutathione concentrations in old TIG-1 cells (PDL 50, 62.5\% of the maximum life span). The enzyme activities in 3 (PDL 62) and 6-wk cultures (PDL 71) are shown by shaded and open columns, respectively. Data are expressed as the means \pm standard deviation $(n=4)$ of three replicate analyses. ${ }^{*} p<0.05$ compared with the control.

remains unchanged (3). Yuan et al. reported that the activity in TIG-3 human diploid cells was in a state of no change (5). Our results are similar to these findings. As shown in Fig. 1-a and b, the SOD activity of the TIG1 cells appeared to be constant during in vitro aging from PDL 44 to 71 , although the activity in young cells decreased with the prolonged culture period. Such a decrease in the SOD activity accompanying the culture period of young cells may be explained in terms of increased cell proliferation and fixed $\mathrm{Cu}$, Zn-SOD activity, 
as described by Kong and Fanburg (23). On the other hand, it has been reported that, during in vitro aging of WI-38 cells, the catalase activity tends to decrease slightly (6) but the GSH-Px activity increases (5). As shown in Fig. 2-a and b, the GSH-Px activity level in old cells (PDL 71) decreased as compared with that in young cells (PDL 44), while the levels in both young and old cells increased with the prolonged culture period. Additionally, the catalase activity of old cells (PDL 71) increased as compared with that of young cells (PDL 44), while those of both young and old cells decreased with increasing culture periods (in vitro aging) as shown in Fig. 3-a and b. Therefore, our results are alike regarding the GSH-Px and catalase activities discussed in previous reports $(5,6)$. Namely, GSH-Px activity increased with increasing culture periods (from PDL 37 to 44 for young cells and from PDL 62 to 71 for old cells), and catalase activity decreased with increasing culture periods (from PDL 37 to 44 and from PDL 62 to 71 , respectively). Slight variations between our results and previous reports may be due either to differences in storage conditions prior to culturing or to different cell strains. As shown in Fig. 4-a and b, the GSH concentrations in young and old cells decreased with increasing culture periods (in vitro aging). Yuan et al. (5) indicated that human diploid cells were more susceptible to oxidative stress at late stages than at early stages, presumably because of the decreases in cellular-GSH concentration and catalase activity. Further, they suggested that GSH might serve as a primary defense against oxidative stress (5). The mechanism of intra-cellular protection against oxidative damage may be low in old cells, in which the damage was greater than in young cells. Conversely, the cellular capacity of protection against oxidative damage may decrease with the prolonged culture period (in vitro aging). Our observation for control cells supports this inference.

We examined the effects of WSP and HC on the TIG-1 cells of different culture ages. The SOD activity in WSPtreated young cells cultured for 4 wk tended to increase as compared with that in the control (Fig. 1-a). The WSP-treated old cells of 6 -wk cultures were virtually equal in SOD activity to the control (Fig. 1-b), implying that WSP treatment might have failed to influence the old cells of PDL 71 ( $89 \%$ of the maximum life span). On the other hand, treatment with WSP or HC caused $10-70 \%$ decreases in SOD activity, except for the WSP treatments for $4 \mathrm{wk}$ in young cells and for $6 \mathrm{wk}$ in old cells, and $\mathrm{HC}$ treatments for $6 \mathrm{wk}$ in young cells and $6 \mathrm{wk}$ in old cells. It can be assumed from these changes in SOD activity that WSP or HC treatment brings about a reduction in oxidative stress since the generation of superoxide radicals in cultured cells is inhibited by WSP (15) or HC (11).

The GSH-Px activity in old cells treated with WSP or $\mathrm{HC}$ for $6 \mathrm{wk}$ was equal to that in the control (Fig. 2-b), whereas the degree of change in young cells after $6 \mathrm{wk}$ was $10-50 \%$ as much in WSP- or HC-treated cells as in control cells (Fig. 2-a). The reason why such a difference occurs between young and old cells is feasibly ex- plained on the assumption that the GSH-Px activity in the old cells (PDL 71) may be enough to detoxify free radicals. In other words, it may be that the GSH-Px activities in old cells without and with WSP or HC treatment are similar to each other or that the stimulatory response of the cells to WSP or HC may have been weakened with age. On the other hand, the GSH-Px activity in young cells was lessened by treatment for $6 \mathrm{wk}$ with WSP or HC. It is conceivable that hydrogen peroxide may have been scavenged by WSP or HC treatment.

Catalase is an important enzyme involved in cell defense against oxygen-mediated cytotoxicity. Catalase activity was increased by WSP or HC treatment except when old cells were treated with HC (Fig. 3-a and b). The elevation of catalase activity in TIG- 1 cells treated with WSP may be attributed to activation of this enzyme in the old cells. The protective mechanisms against oxidative damage may not be sufficiently functional to obstruct damage in the old cells, as is possible in young cells. Above all, the catalase activity level is known to decrease with in vitro aging. The decreased antioxidative capability of old cells acts as a trigger for aging and cancer. Therefore, the activation of catalase by WSP treatment of old cells may lessen such risk factors.

GSH scavenges free radicals directly, or it is involved in the radical scavenging pathway as a substrate for GSH-Px. WSP elevated the level of GSH in the TIG-1 cells except when treating young cells for $4 \mathrm{wk}$ (Fig. 4-a and $b$ ). It thus seems conceivable that WSP treatment may exert some influence on intracellular radical density with the intervention of GSH.

WSP treatment may positively affect the suppression of fibroblast senescence. This finding invites speculation that WSP can improve cellular protection against oxidative damage.

\section{Acknowledgments}

We thank Ms. W. Miyata for her critical review of the manuscript. We also thank Dr. H. Kondo for supplying us with the human diploid fibroblasts, TIG-1. This work was supported by a Grant-in-Aid for Scientific Research from the Ministry of Education, Science, Sports and Culture, Japan.

\section{REFERENCES}

1) Harman D. 1956. Aging: a theory based on free radical and radiation chemistry. J Gerontol 11: 298-300.

2) Harman D. 1981. The aging process. Proc Natl Acad Sci USA 78: 7124-7128.

3) Yamanaka N, Deamer D. 1974. Superoxide dismutase activity in WI-38 cell cultures: Effects of age, trypsinization, and SV-40 transformation. Physiol Chem Phys 6: 95-106.

4) Duncan NR, Dellorco RT, Kirk KD. 1979. Superoxide dismutase specific activities in cultured human diploid cells of various donor age. J Cell Physiol 98: 437-442.

5) Yuan H, Kaneko T, Matsuo M. 1996. Increased susceptibility of late passage human diploid fibroblasts to oxidative stress. Exp Gerontol 31: 465-474.

6) Sun AS, Aggarwal BB, Packer L. 1975. Enzyme levels of 
normal human cells: Aging in culture. Arch Biochem Biophys 170: 1-11.

7) Mbemba F, Houbin A, Raes M, Remacle J. 1985. Subcellular localization and modification with ageing of glutathione, glutathione peroxidase and glutathione reductase activities in human fibroblasts. Biochim Biophys Acta 838: 211-220.

8) Tappel AI. 1978. Glutathione peroxidase and hydroperoxides. Methods Enzymol 52: 506-513.

9) Cristofalo VJ. 1972. Animal cell cultures as a model system for the study of aging. Adv Geront Res 4: 45-79.

10) Fulder SJ. 1977. The growth of cultured human fibroblasts treated with hydrocortisone and extracts of the medicinal plant Panax Ginseng. Exp Geront 12: 125131.

11) Korchak HM, Weissmann G. 1978. Changes in membrane potential of human granulocytes antecede the metabolic responses to surface stimulation. Proc Natl Acad Sci USA 75: 3818-3822.

12) Lowlor SM, O'Brien NM. 1995. Astaxanthin: Antioxidant effects in chicken embryo fibroblasts. Nutr Res 15: 1695-1704.

13) Leist M, Raab B, Maurer S, Rösick U, Brigelius-Flohé R. 1996. Conventional cell culture media do not adequately supply cells with antioxidants and thus facilitate peroxide-induced genotoxicity. Free Radic Biol Med 21: 297-306.

14) Keogh BP, Tresini M, Cristofalo VJ, Allen RG. 1996. Effects of cellular aging on the induction of $c$-fos by antioxidant treatments. Mech Ageing Dev 86: 151-160.
15) Okada Y, Okada M. 1998. Scavenging effect of water soluble proteins in broad beans on free radicals and oxygen species. J Agrical Food Chem 46: 401-406.

16) Okada Y, Okada M. 1999. Increase of the cellular growth of old human diploid fibroblasts by radical scavenger: water-soluble protein from broad beans. Gerontology 45: 72-78.

17) Okada Y, Kimura H, Aoyama T. 1986. Neurotropin increases in vitro life span of human fibroblasts. Mech Ageing Dev 35: 133-143.

18) Rikans LE, Snowden CD, Moore DR. 1992. Effect of aging on enzymatic antioxidant defenses in rat liver mitochondria. Gerontology 38: 133-138.

19) Crapo JD, McCord JM, Fridovich I. 1978. Preparation and assay of superoxide dismutases. Methods Enzymol 53: 382-393

20) Chance B, Maehly AC. 1955. Assay of catalases and peroxidases. Methods Enzymol 2: 764-775.

21) Tietze F. 1969. Enzymatic method for quantitative determination of nanogram amounts of total and oxidized glutathione: Application to mammalian blood and other tissues. Analyt Biochem 27: 502-522.

22) Bradford MM. 1976. A rapid and sensitive method for the quantitation of microgram quantities of protein utilizing the principle of protein-dye binding. Analyt Biochem 72: 248-254.

23) Kong X-J, Fanburg BL. 1992. Regulation of $\mathrm{Cu}, \mathrm{Zn}$ superoxide dismutase in bovine pulmonary artery endothelial cells. J Cell Physiol 153: 491-497. 